Are meningeal cells also involved in specific homing of ALL cells into the CNS? Is adhesion-mediated chemoresistance of ALL cells in contact with meningeal cells simply a cause of decreased proliferation and therefore diminished vulnerability to chemotherapeutic agents or is an active process involved (e.g., regulation of drug transporters)? Furthermore, it would be interesting to determine whether adhesion capacity of ALL cells to the CNS microenvironment could be used to improve CNS diagnostics. A recent report suggests that surface expression of $\alpha 5$-integrins on ALL cells is associated with the number of ALL cells in the cerebrospinal fluid detectable by diagnostic lumbar puncture. ${ }^{18}$ Finally, there is a need to consider that mobilizing dormant ALL cells by breaking adhesive bonds with meningeal cells may also confer potential risks. Re-awakening leukemic cells may cause a resumption of proliferation and therefore overt CNS disease, an aspect which will have to be clarified in the future.

The recent study by Jonart et al. shapes a sharper image of the complex mechanisms of both CNS infiltration and CNS relapse, and may ultimately contribute to improved strategies for targeted treatment of CNS leukemia in ALL.

\section{References}

1. Pui CH, Howard SC. Current management and challenges of malignant disease in the CNS in paediatric leukaemia. Lancet Oncol. 2008;9(3):257-268

2. Cheung YT, Khan RB, Liu W, et al. Association of cerebrospinal fluid biomarkers of central nervous system injury with neurocognitive and brain imaging outcomes in children receiving chemotherapy for acute lymphoblastic leukemia. JAMA Oncol. 2018;4(7):e180089.

3. Lenk L, Alsadeq A, Schewe DM. Involvement of the central nervous system in acute lymphoblastic leukemia. Opinions on molecular mechanisms and clinical implications based on recent data. Cancer Metastasis Rev. 2020;39(1):173-187

4. Frishman-Levy L, Shemesh A, Bar-Sinai A. et al. Central nervous sys- tem acute lymphoblastic leukemia: role of natural killer cells. Blood. 2015;125(22):3420-3431.

5. Jonart LM, Ebadi M, Basile P, et al. Disrupting the leukemia niche in the central nervous system attenuates leukemia chemoresistance. Haematologica. 2020;105(8):2130-2140.

6. Williams MTS, Yousafzai YM, Elder A, et al. The ability to cross the blood-cerebrospinal fluid barrier is a generic property of acute lymphoblastic leukemia blasts. Blood. 2016;127(16):1998-2006.

7. Price RA. Histopathology of CNS leukemia and complications of therapy. Am J Pediatr Hematol Oncol. 1979;1(1):21-30.

8. Basile P, Jonart LM, Ebadi M, Johnson K, Kerfeld M, Gordon PM. The meninges enhance leukaemia survival in cerebral spinal fluid. Br J Haematol. 2020;189(3):513-517.

9. Aguirre-Ghiso JA. Models, mechanisms and clinical evidence for cancer dormancy. Nat Rev Cancer. 2007;7(11):834-846.

10. Kato I, Nishinaka Y, Nakamura M, et al. Hypoxic adaptation of leukemic cells infiltrating the CNS affords a therapeutic strategy targeting VEGFA. Blood. 2017;129(23):3126-3129.

11. Krause S, Pfeiffer C, Strube S, et al. Mer tyrosine kinase promotes the survival of $t(1 ; 19)$-positive acute lymphoblastic leukemia (ALL) in the central nervous system (CNS). Blood. 2015;125(5):820-830.

12. Yao H, Price TT, Canelli G, et al. Leukaemia hijacks a neural mechanism to invade the central nervous system. Nature. 2018;560(7716):55-60

13. Buonamici S, Trimarchi T, Ruocco MG, et al. CCR7 signalling as an essential regulator of CNS infiltration in T-cell leukaemia. Nature. 2009:459(7249):1000-1004

14. Ebinger S, Özdemir EZ, Ziegenhain C, et al. Characterization of rare, dormant, and therapy-resistant cells in acute lymphoblastic leukemia. Cancer Cell. 2016;30(6):849-862 .

15. Alsadeq A, Fedders H, Vokuhl C, et al. The role of ZAP70 kinase in acute lymphoblastic leukemia infiltration into the central nervous system. Haematologica. 2017;102(2):346-355.

16. Chen J, Carey K, Godowski PJ. Identification of Gas6 as a ligand for Mer, a neural cell adhesion molecule related receptor tyrosine kinase implicated in cellular transformation. Oncogene. 1997;14(17):20332039

17. Zhang J, Ren X, Shi W, et al. Small molecule Me6TREN mobilizes hematopoietic stem/progenitor cells by activating MMP-9 expression and disrupting SDF-1/CXCR4 axis. Blood. 2014;123(3):428-441.

18. Shah Scharff BFS, Modvig S, Thastrup M, et al. A comprehensive clinical study of integrins in acute lymphoblastic leukemia indicates a role of $\alpha 6 / C D 49 f$ in persistent minimal residual disease and $\alpha 5$ in the colonization of cerebrospinal fluid. Leuk Lymphoma. $2020 \mathrm{Feb}$ 28;1-5. [Epub ahead of print]

\title{
BCL2 dependency in diffuse large B-cell lymphoma: it's a family affair
}

\author{
Shannon M. Matulis and Lawrence H. Boise
}

Department of Hematology and Medical Oncology Emory School of Medicine and the Winship Cancer Institute, Emory University; Atlanta, GA, USA

E-mail: LAWRENCE H. BOISE - lboise@emory.edu

doi:10.3324/haematol.2020.253591

$\mathrm{D}$ iffuse large B-cell lymphoma (DLBCL) is the most common form of non-Hodgkin lymphoma, accounting for approximately $25 \%$ of all lymphomas. ${ }^{1}$ DLBCL is highly heterogeneous, so responses to standard therapy, R-CHOP (rituximab plus cyclophosphamide, doxorubicin, vincristine, and prednisone) are mixed. ${ }^{2}$ The response, as well as mechanisms of resistance to therapy, are associated with a cell's apoptotic threshold. ${ }^{3}$ Therefore, determining the molecular basis for a tumor's ability to survive can provide insights into drug resistance as well as opportunities for precision medicine. In this issue of Haematologica, Smith et al. demonstrate the importance of the BCL2 family of anti-apoptotic proteins BCL2, BCLXL, and MCL1 in the survival of DLBCL, potentially revealing new treatment strategies. ${ }^{4}$

Inappropriate activation of oncogenes can result in cell death through the activation of pro-apoptotic proteins of the BCL2 family. Therefore, to survive the transformation process, tumor cells become more dependent on their anti-apoptotic BCL2 proteins (e.g., BCL2, BCLXL, and MCL1) than their normal counterparts. ${ }^{5.7}$ This dependency is the result of binding and neutralizing the pro-apoptotic family members (e.g., BIM, BAK, and BAX) and is often referred to as mitochondrial priming, as increased 
priming results in a lower apoptotic threshold..$^{5-7}$ Thus priming of anti-apoptotic BCL2 family proteins in cancer leads to increased sensitivity to therapy, as well as making BCL2 proteins excellent targets for therapy. ${ }^{5-7}$

Venetoclax (ABT-199) is a potent and selective inhibitor of BCL2. ${ }^{8}$ The importance of BCL2 in DLBCL is well established. The $t(14 ; 18)$ translocation, occurring in approximately $20 \%$ of DLBCL, juxtaposes BCL2 to the immunoglobulin heavy chain gene enhancers resulting in overexpression. ${ }^{2}$ While this translocation is most frequently found in the germinal center B-cell subtype of DLBCL, amplification and transcriptional upregulation of BCL2 are typically found in the activated B-cell molecular subtype..$^{10}$ Despite this, the clinical success of venetoclax in DLBCL has been disappointing, with an overall response rate of only $18 \%$, regardless of BCL2 expression level. ${ }^{11}$

These findings led Smith et al. to propose a role for other anti-apoptotic proteins in DLBCL survival. To investigate this possibility, they employed venetoclax as well as A-1331852 and S63845, selective inhibitors for BCLXL and MCL1, respectively, as tools to determine the role of each anti-apoptotic protein in DLBCL survival. Using primary cells isolated from patients' samples, as well as a panel of cell lines representing the main subtypes of DLBCL, they demonstrated that all three inhibitors displayed activity in both the patients' samples and the cell lines. About half of the cell lines were preferentially sensitive to only one inhibitor, suggesting sole dependence on that anti-apoptotic family member for survival.

However, four of the 18 cell lines and at least two of the seven patients' samples showed sensitivity to two inhibitors, suggesting co-dependence on more than one anti-apoptotic protein for survival, a characteristic reported in other hematologic malignancies. ${ }^{12}$ Resistance to all three mimetics was seen in six of the 18 cell lines; however, the mechanism of resistance was not explored. To further support a role for MCL1 and BCLXL in DLBCL the authors showed that silencing the anti-apoptotic proteins with short interfering (si)RNA was sufficient to induce apoptosis in cell lines sensitive to S63845 and A1331852, respectively.

Based on the sensitivity data, the authors determined the expression levels and ratios of the BCL2 proteins and found that expression was highly variable between cell lines. Concluding that expression alone could not account for sensitivity to the inhibitors they moved on to examine protein interactions. Previous studies in other hematologic malignancies had demonstrated that the binding pattern of pro-apoptotic proteins to anti-apoptotic proteins is also a predictor of sensitivity to these small molecule

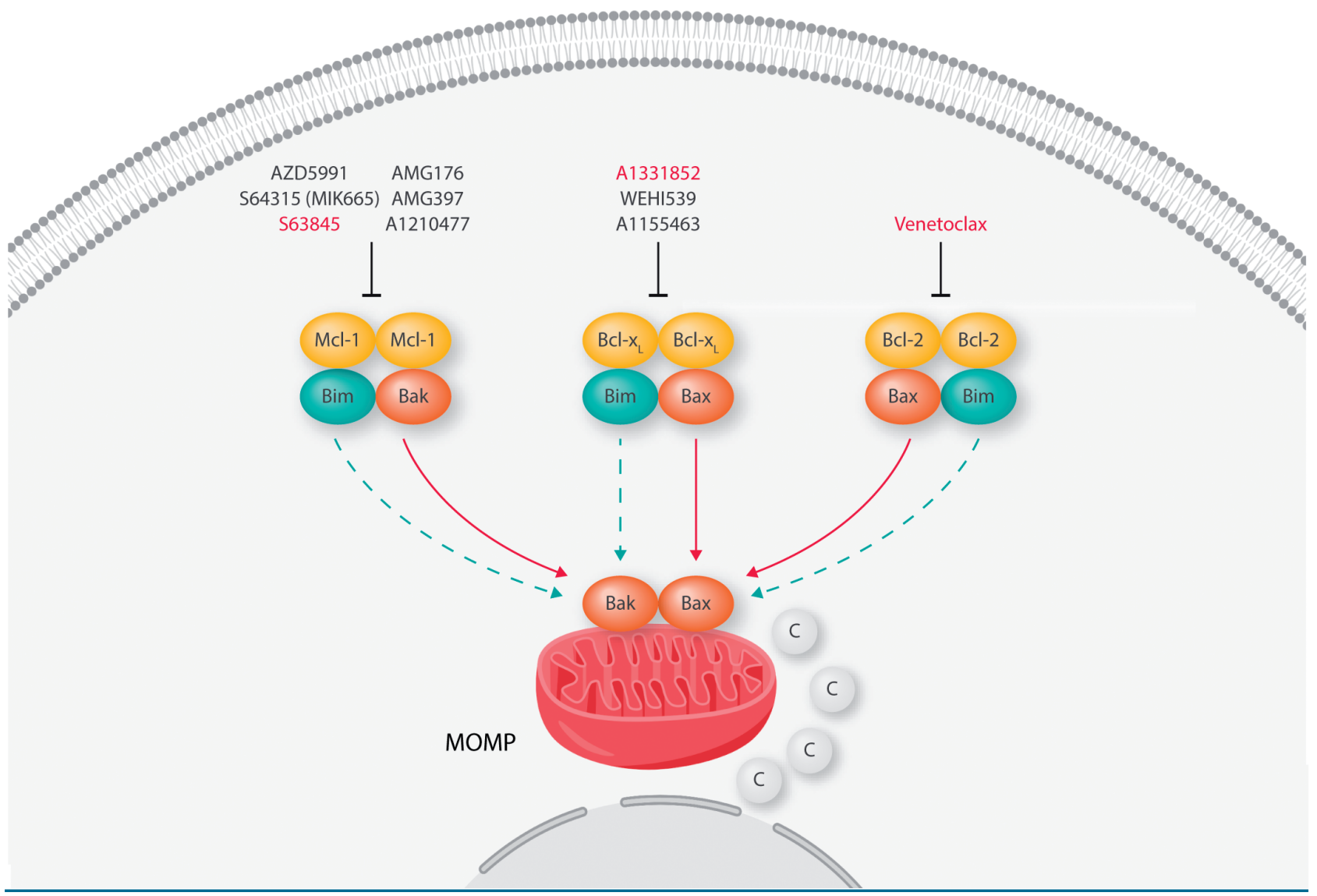

Figure 1. Schematic of the mechanism of action of selective BCL2 family inhibitors. Inhibitors disrupt interactions between anti-apoptotic BCL2 family members (green) and either the BH3-only activator protein BIM (orange) or the pro-apoptotic effectors BAX and BAK (red). This results in the release of activated effectors (solid arrows) or BIM, which can activate the effectors (dashed arrows) resulting in mitochondrial outer membrane permeabilization (MOMP) and cytochrome $\mathrm{C}$ release. The inhibitors in red were the ones used in the study by Smith et al. ${ }^{4}$ 
inhibitors, and this was found to be true in DLBCL as well. ${ }^{12}$ Using co-immunoprecipitation, the authors showed BIM and BAX bound to BCL2 in BCL2-depen$\mathrm{dent} / \mathrm{venetoclax-sensitive} \mathrm{cell} \mathrm{lines} \mathrm{and} \mathrm{BAX} \mathrm{release} \mathrm{upon}$ drug treatment. In BCLXL-dependent/A-1331852-sensitive DLBCL lines, BIM, BAX, and BAK were bound to BCLXL and all three were released following treatment. The pro-apoptotic proteins BIM and BAK were bound to MCL1 and both displaced upon treatment with S63845 in MCL1-dependent cell lines.

BCL2 family expression data in the six resistant lines showed that five expressed some degree of all three antiapoptotic proteins and one line expressed two. Furthermore, all six lines expressed BAK and/or BAX, with some variability in BIM expression. Co-immunoprecipitation assays performed in two of the resistant lines showed BIM binding to at least one anti-apoptotic protein but minimal binding of BAX or BAK. Displacement data following drug treatment in all six lines are necessary to gain a better understanding of the protein interactions driving this resistance. However, it remains unclear how a cell could be resistant to all three inhibitors. One possibility is that resistant cells utilize more than one BCL2 family member, requiring multiple inhibitors to be used in combination. Alternatively, the cells could be dependent on an anti-apoptotic BCL2 protein that was not tested. For example, BCL-w was recently reported to be overexpressed in DLBCL and investigating the role of this anti-apoptotic protein or that of BCL2A1 (A1/Bfl1) may reveal that it is implicated in cell survival in DLBCL.13 While there is not currently a selective inhibitor for BCL$\mathrm{W}$, testing cell lines that are resistant to venetoclax and A1331852 with ABT-737, which was reported to inhibit $\mathrm{BCL}-\mathrm{w}$, may provide indirect evidence of dependency. ${ }^{14}$

The authors used both siRNA and CRISPR to discern the contribution of the displaced proteins to the initiation of apoptosis. BIM is necessary for S63845-induced apoptosis in the two cell lines tested, whereas its role in venetoclax- and A-1331852-induced apoptosis is cell-line dependent. BAK and BAX are involved to some degree in the apoptotic response to all three inhibitors, however the contribution of each in initiation versus amplification of the apoptotic signal is not entirely clear.

The authors suggest that activated BAX released from BCL2 directly activates BAK; however, previous studies indicate that $\mathrm{BAX}$ is a poor activator of $\mathrm{BAK} \cdot{ }^{15}$ An alternative explanation would be that displaced BIM is responsible for activating BAK. In the two BCLXL-dependent cell lines examined only one was protected from A-1331852induced apoptosis when BAX was silenced and the apoptotic response was not altered in either in response to the silencing of BAK. Furthermore, CRISPR knockout of BAK appeared to have a minor, A-1331852 dose-independent, effect on apoptosis in the one cell line shown. Repeating these experiments on a larger panel of cell lines is necessary to understand the relative importance of these proteins in A-1331852-induced apoptosis. The BAX siRNA data support the authors' assertion that BAX is required for S63845-induced apoptosis; however, it remains unclear what protein(s) released from MCL1 are required to activate BAX. It should be noted that incomplete silencing of genes occurred in some experiments, which could influence the interpretation of the results. Performing the mechanistic experiments with CRISPR knockouts of BIM and BAX, along with BAK, would provide further insight into the role of each of these proteins in BH3-mimetic-induced apoptosis in DLBCL. Regardless, the studies clearly point to the importance of several BCL2 family members in the survival of DLBCL cells and provide insights into a potential means of targeting these vulnerabilities.

The potential of ex vivo testing as a means to deliver precision medicine based on functional testing instead of genotype has been reported in multiple myeloma with venetoclax and also in acute myeloid leukemia. ${ }^{16,17}$ Given the data presented here, one could envisage a way this type of assay could be used in DLBCL. While venetoclax was not effective as a sole agent in DLBCL, it is being tested in combination with current therapeutics. Recently published data from the CAVALLI phase Ib trial demonstrate the benefit of combining venetoclax with R-CHOP or GCHOP (obinutuzumab plus cyclophosphamide, doxorubicin, vincristine, and prednisone) in BCL2-positive, MYCpositive DLBCL, with seven of eight patients $(87.5 \%)$ reaching complete remission. ${ }^{18}$ Additionally, a clinical trial evaluating the MCL1 inhibitor S64315 (MIK665) in MYCpositive DLBCL is currently recruiting (NCT02992483). ${ }^{19}$ Ex vivo testing of patients prior to therapy initiation or enrollment on a clinical trial could provide guidance on treatment and spare the patient from ineffective therapy.

\section{References}

1. Freedman AS, Jacobson CA, Ng A, Aster JC. Non-Hodgkin lymphoma. In: DeVita, Hellman, and Rosenberg's Cancer Principles \& Practice of Oncology. 11th Edition, 2019. pp 1671-1707. Editors: DeRita VT Jr, Lawrence TS, Rosenberg SA. Published by Wolters Kluwer.

2. Liu Y, Barta SK. Diffuse large B-cell lymphoma: 2019 update on diagnosis, risk stratification, and treatment. Am J Hematol. 2019;94(5):604-616.

3. Ni Chonghaile T, Sarosiek KA, Vo TT, et al. Pretreatment mitochondrial priming correlates with clinical response to cytotoxic therapy. Science. 2011;334(6059):1129-1133.

4. Smith VM, Dietz A, Henz K, et al. Specific interactions of BCL-2 family proteins mediate sensitivity to $\mathrm{BH} 3$-mimetics in diffuse large B-cell lymphoma. Haematologica. 2020;105(8):2150-2163.

5. Certo M, Del Gaizo Moore V, Nishino M, et al. Mitochondria primed by death signals determine cellular addiction to antiapoptotic BCL2 family members. Cancer Cell. 2006;9(5):351-365.

6. Chipuk JE, Moldoveanu T, Llambi F, Parsons MJ, Green DR. The BCL2 family reunion. Mol Cell. 2010;37(3):299-310.

7. Davids MS, Letai A. Targeting the B-cell lymphoma/leukemia 2 family in cancer. J Clin Oncol. 2012;30(25):3127-3135.

8. Souers AJ, Leverson JD, Boghaert ER, et al. ABT-199, a potent and selective BCL2 inhibitor, achieves antitumor activity while sparing platelets. Nat Med. 2013;19(2):202-208.

9. Willis TG, Dyer MJ. The role of immunoglobulin translocations in the pathogenesis of B-cell malignancies. Blood. 2000;96(3):808-822.

10. Iqbal J, Neppalli VT, Wright G, et al. BCL2 expression is a prognostic marker for the activated B-cell-like type of diffuse large B-cell lymphoma. J Clin Oncol. 2006;24(6):961-968.

11. Davids MS, Roberts AW, Seymour JF, et al. Phase I first-in-human study of venetoclax in patients with relapsed or refractory nonHodgkin lymphoma. J Clin Oncol. 2017;35(8):826-833.

12. Morales AA, Kurtoglu M, Matulis SM, et al. Distribution of Bim determines MCL1 dependence or codependence with BCLXL/BCL2 in MCL1-expressing myeloma cells. Blood. 2011;118(5):1329-1339.

13. Adams CM, Mitra R, Gong JZ, Eischen CM. Non-Hodgkin and Hodgkin lymphomas select for overexpression of BCLW. Clin Cancer Res. 2017;23(22):7119-7129.

14. Oltersdorf T, Elmore SW, Shoemaker AR, et al. An inhibitor of BCL2 
family proteins induces regression of solid tumours. Nature. 2005;435(7042):677-681.

15. Iyer S, Uren RT, Dengler MA, et al. Robust autoactivation for apoptosis by BAK but not BAX highlights BAK as an important therapeutic target. Cell Death Dis. 2020;11(4):268.

16. Matulis SM, Gupta VA, Neri P, et al. Functional profiling of venetoclax sensitivity can predict clinical response in multiple myeloma. Leukemia. 2019;33(5):1291-1296.
17. Swords RT, Azzam D, Al-Ali H, et al. Ex-vivo sensitivity profiling to guide clinical decision making in acute myeloid leukemia: a pilot study. Leuk Res. 2018;64:34-41.

18. Zelenetz AD, Salles G, Mason KD, et al. Venetoclax plus R- or G$\mathrm{CHOP}$ in non-Hodgkin lymphoma: results from the CAVALLI phase 1 b trial. Blood. 2019;133(18):1964-1976.

19. Adams CM, Clark-Garvey S, Porcu P, Eischen CM. Targeting the BCL2 family in B cell lymphoma. Front Oncol. 2019;8:636.

\title{
Insights into vitamin K-dependent carboxylation: home field advantage
}

\author{
Francis Ayombil ${ }^{1}$ and Rodney M. Camire ${ }^{1,2}$ \\ ${ }^{1}$ Division of Hematology and the Raymond G. Perelman Center for Cellular and Molecular Therapeutics, The Children's Hospital of \\ Philadelphia and ${ }^{2}$ Department of Pediatrics, Perelman School of Medicine, University of Pennsylvania, Philadelphia, PA, USA \\ E-mail: RODNEY M. CAMIRE - rcamire@pennmedicine.upenn.edu
}

doi:10.3324/haematol.2020.253690

$\mathrm{V}$ itamin K-dependent (VKD) proteins play critical roles in blood coagulation, bone metabolism, and other physiologic processes. These proteins undergo a specific post-translational modification called gamma $(\gamma)$-carboxylation which is critical to their biologic function. ${ }^{1}$ The reaction, which occurs in the endoplasmic reticulum (ER) and requires reduced vitamin $\mathrm{K}$, carbon dioxide and oxygen as co-factors, is catalyzed by $\gamma$-glutamyl carboxylase (GGCX). GGCX converts several glutamic acid residues (Glu) on its protein substrate [e.g. prothrombin, FVII, FIX, FX, PC, PS, PZ, and bone Gla protein (BGP)] to $\gamma$-carboxy-glutamic acid, otherwise known as Gla. ${ }^{2}$ How does this enzyme pick its protein substrate and modify specific glutamic acid residues? In work spanning over 30 years, researchers identified a critical sequence called the propeptide region that is $\mathrm{N}$-terminal to the mature protein (Figure 1). GGCX binds the propeptide and directs carboxylation of 9-13 Glu residues on the socalled Gla domain in a processive fashion. ${ }^{2}$ The signal sequence and propeptide region are removed by peptidases prior to secretion of the mature VKD protein (Figure 1). For the VKD coagulation factors, the enhanced net negative charge following carboxylation in the Gla domain allows for high affinity divalent metal ion binding. ${ }^{3}$ This changes the structural conformation of the Gla domain which facilitates binding to anionic phospholipids and localizes these proteins to the site of vascular injury. ${ }^{3,4}$ Defects of VKD protein carboxylation cause bleeding disorders, and inhibition of this pathway is the basis of warfarin anticoagulation. ${ }^{2}$

Acquiring mechanistic information about GGCX and deciphering how the propeptide influences carboxylation has been challenging. Since GGCX is an integral membrane ER protein (Figure 1), extracting it in a functional state is difficult and requires artificial conditions to study it. Early work used crude microsomal extracts or detergent-solubilized liver microsomes following warfarin treatment or vitamin K-deficient animals which contained the enzyme and small amounts of endogenous protein substrate (e.g. prothrombin). ${ }^{1}$ Advancements to this system incorporated artificial peptide substrates for GGCX such as FLEEL (residues 5-9 of rat prothrombin). ${ }^{5}$ In the late 1980s, it was recognized that the propeptide sequence is critical for VKD protein carboxylation. ${ }^{6}$ This insight led to the development of GGCX substrates that contained a propeptide sequence and portions of the Gla domain which are superior when compared to FLEEL alone. ${ }^{7,8}$ These and other substrates have been used to demonstrate the importance of propeptide affinity in substrate recognition using either crude preparations or purified forms of GGCX and increased our understanding about the enzyme. ${ }^{9}$ Further insights into the importance of the propeptide came from studies using mutant peptides and identification of naturally occurring mutations in the propeptide region of FIX. ${ }^{10,11}$ However, this knowledge about the function of GGCX was obtained outside of its natural environment under artificial conditions. To better understand VKD carboxylation in its native milieu, Tie and Stafford developed a cell-based reporter assay to study $\gamma$-carboxylation and the entire VKD cycle. ${ }^{12}$ In this system, a chimeric reporter-protein, FIXgla-PC is used, in which the PC backbone was replaced at the N-terminus with the FIX Gla domain. ${ }^{12,13}$ This allowed for an ELISA-based quantification of carboxylated reporter protein using a capture antibody that recognizes only a fully carboxylated FIX Gla domain and an antibody against PC. The advantage of the system is that it allows for functional assessment of the VKD cycle enzymes, including GGCX, in an environment that requires the enzymes to interact with their physiologic substrates, a departure from systems previously employed.

In this issue of Haematologica, Hao et al. use this cellbased assay to study the role of the propeptide in directing carboxylation of VKD proteins. ${ }^{14}$ Previous studies indicate that the propeptide region of VKD coagulation factors show considerable variation in their affinities for GGCX with FX, FIX and PC showing high $\left(\mathrm{K}_{\mathrm{d}} \sim 1 \mathrm{nM}\right)$, intermediate $\left(\mathrm{K}_{\mathrm{d}} \sim 5 \mathrm{nM}\right)$, and low affinity $\left(\mathrm{K}_{\mathrm{d}} \sim 20 \mathrm{nM}\right)$, respectively (Figure 1). ${ }^{15}$ It is thought that these disparate affinities contribute to the heterogeneity in carboxylation in mammalian expression systems. Furthermore, it is thought that there is likely an optimal propeptide affinity that best directs carboxylation. To better understand how GGCX interacts with its protein substrates via propeptide binding in its natural environment, the authors created a series of chimeric 